\title{
FÍSICA Y CULTURA CIENTÍFICA EN LA EDUCACIÓN MEDIA: REVISIÓN CRÍTICA DEL CURRÍCULUM EN CIENCIAS NATURALES
}

\section{PHYSICS AND SCIENTIFIC CULTURE IN SECONDARY EDUCATION: CRITICAL REVIEW OF THE CURRICULUM IN NATURAL SCIENCES}

Heriberto Manzanilla*, Nelson Falcón**

\section{Resumen}

Los avances de la tecnología invitan a la "alfabetización científica" durante la escolaridad obligatoria. Se presenta una metodología para organizar los contenidos curriculares en el área de ciencias naturales, empleando la episteme de la física con la finalidad de evaluar la cultura científica. Se analizan los contenidos en la asignatura de física, como paradigma de las ciencias naturales, en el currículum de educación media en Chile, España, México y Colombia. Los resultados muestran diferencias entre el currículum prescrito y el implementado, en cuanto a contenidos y alcances para fomentar una cultura científica a nivel medio. Se concluye que los contenidos de la asignatura de física no son suficientes para la comprensión de los fenómenos naturales y de la tecnología, tanto por la completitud, como por la carencia de orden conceptual. Se sugieren alternativas para mejorar la enseñanza de la física y la formación de la cultura científica a nivel de educación media.

\section{Palabras Claves:}

Evaluación de la educación, enseñanza de ciencias básicas, cultura científica, enseñanza de la física, política educacional, educación comparada.

* Magister en Educación en Física. Docente de enseñanza media, colegio Santa Elena College, Coquimbo, Chile. email: mrheriberto.educ@gmail.com, ORCID 0000-0003-0077-8464

** Dr. en Física. Docente Universidad de Carabobo, Facultad Experimental de Ciencias y Tecnología, Dpto. de Física. Valencia, Venezuela. email: nfalcon@uc.edu.ve, ORCID 00000001-5286-5047 


\begin{abstract}
Advances in technology invite "scientific literacy" during compulsory schooling. A methodology is presented to organize the curricular contents in the area of natural sciences, using the episteme of physics in order to evaluate scientific culture. The contents in the subject of physics, as a paradigm of the natural sciences, in the secondary education curriculum in Chile, Spain, Mexico and Colombia are analyzed. The results show differences between the prescribed and implemented curriculum in terms of content and scope to foster a scientific culture at the secondary level. It is concluded that the contents of the subject of physics are not enough for the understanding of natural phenomena and technology, both because of the completeness, and the lack of conceptual order. Alternatives are suggested to improve the teaching of physics and the education of scientific culture at secondary school level.
\end{abstract}

\title{
Keywords
}

Evaluation of education, teaching of fundamental sciences, scientific culture, teaching of physics, educational policy, comparative education.

\section{Introducción}

El vertiginoso avance de la tecnología en la vida cotidiana, principalmente desde mediados del siglo pasado, y que caracteriza a la llamada postmodernidad, ha impulsado la necesidad del manejo de información científica-tecnológica para el ciudadano común, al punto que es casi de perogrullo referirse a la necesidad de una formación básica en ciencias naturales en los currículums de la educación básica, media y preuniversitaria.

La "alfabetización científica", es un término genérico para invocar la necesidad del dominio de aspectos básicos de las ciencias naturales y de las tecnologías que de ellas se derivan, en los ciudadanos en general, y más específicamente para los estudiantes de la escolaridad obligatoria (Sasseron y Carvalho, 2011; Gil-Pérez y Vilches-Peña, 2001). Por otro lado, la Organización para la Cooperación y el 
Desarrollo Económico (OCDE), a través del Programme for International Student Assessement (PISA), define la alfabetización científica como:

"La capacidad de un individuo de utilizar el conocimiento cientifco para identificar preguntas, adquirir nuevos conocimientos, explicar fenómenos cientificos y sacar conclusiones basadas en evidencias respecto de temas relativos a la ciencia, comprender los rasgos especificos de la ciencia como una forma de conocimiento y búsqueda humana, ser consciente de cómo la ciencia y tecnología dan forma a nuestro mundo material, intelectual y cultural, y tener la voluntad de involucrarse en temas relativos a la ciencia y con ideas científicas, como un ciudadano reflexivo" (OCDE, 2009, p. 128).

En un sentido amplio en idioma español se utiliza el término "alfabetización científica" como la enseñanza que promueve capacidades y competencias que permiten a los estudiantes interpretar los fenómenos naturales o las aplicaciones tecnológicas, mediante modelos científicos; casi con el mismo significado que en la lengua francesa: "Alphabétisation Scientifique" (Fourez, 1996); y que los Anglosajones quienes lo refieren genéricamente como "Scientific Literacy”, con una acepción muy variada (Laugksch,2000). En idioma portugués los autores prefieren distinguir (Gómez, 2012) entre letramiento científico, en el sentido de escribir o "hacer" publicaciones científicas por sus métodos o discursos, alfabetización cientifica como las capacidades de pensamiento organizado y riguroso (pensamiento científico) y enculturación cientifica, referida al proceso de asimilación de nociones, ideas y conceptos científicos como parte de sus vivencias, de modo análogo a las componentes de la cultura individual, tanto históricas como sociales y religiosas.

De lo anterior se comprende que no necesariamente la finalidad de la enseñanza de las ciencias naturales en un currículum escolar obligatorio, para todas las individualidades, requiera de una alfabetización científica ni de un letramiento científico, puesto que la ciencia (en el sentido de ciencias naturales) es solo uno, entre los posibles enfoques, de percepción de la realidad. En concreto, un músico, un artista, un futuro empresario o un religioso no necesariamente requieren de un pensamiento lógico-sistemático y contrastable 
como los científicos, ni el lenguaje discursivo de las ciencias naturales (metalenguaje matemático, por ejemplo).

Sin embargo, todos los seres humanos estamos sujetos a los fenómenos, procesos e interacciones con la naturaleza, por lo tanto el conocer la fenomenología y las "leyes" que gobiernan la naturaleza es, no solo útil, sino prioritario para la sobrevivencia, y en la sociedad postmoderna donde coexistimos rodeados de ingenios, artefactos y máquinas (tecnologías), que son aplicaciones del conocimiento científico de dichas "leyes" naturales, requerimos de una mínima enculturización cientifica sobre la naturaleza (physica), para desenvolvernos adecuadamente en la vida cotidiana, sea este el ambiente natural, rural o citadino. Un currículum escolar debe entonces dar cuenta de esa cultura científica sobre las regularidades de la naturaleza y sus fenómenos. El término enculturización proviene del inglés enculturation, que denota el proceso de integración sociocultural de un individuo con su entorno (Collins y Bielaczyc, 1999).

La cultura científica sobre la naturaleza, vale decir la enculturización en física, debe permitir, entre otros aspectos, analizar los fenómenos naturales y sus implicaciones, manejar conceptos básicos y generalidades fundamentales de la ciencia, estimular la objetividad, el pensamiento crítico, reflexivo y creativo.

Surge entonces la inquietud sobre la elección de los contenidos, su secuencia y alcances, y la interrogante sobre cuál ha de ser el enfoque didáctico para esa visión holística, fenomenológica y conceptual de la naturaleza (physica). Particularmente, sobre la enculturización científica en los jóvenes y adolescentes, a partir de los doce años, que es la etapa donde el educando va construyendo progresivamente un pensamiento abstracto (Thagard 1992, Orellana-Sepúlveda et al 2018), se plantean preocupaciones desde el punto de vista cognitivo, relacionadas con interrogantes que demandan explicaciones racionales de los hechos, fenómenos y procesos de la realidad. Por ello es menester dilucidar qué y cómo enseñar física en la educación media o secundaria, cuando el joven es capaz de intuir, adivinar o deducir situaciones a partir de la observación y tiene el potencial de manejar eficazmente su propio pensamiento y de aprender en sentido amplio, de sí mismo y de su entorno. En el 
entendido que tal educación secundaria en física debe responder también a las necesidades de contenidos pertinentes para la vida, esto en el sentido de las premisas de "Los Siete Saberes Necesarios para la Educación del Futuro" de Edgar Morín (Morín, 1999, Arias y Navarro, 2017, Rodríguez 2017), con una secuencia que posibilite el aprendizaje significativo (Ausubel et al, 1983; Thagard 1992) y un enfoque que permita la transposición didáctica (Chevallard, 1998; Ruiz 2007).

Para ello, analizamos la episteme de la física, como disciplina autónoma en la sección 2, con la finalidad de estudiar la concatenación de ideas y conceptos de las teorías y modelos para la comprensión de los fenómenos naturales. Todo ello como fundamento para proponer una selección de contenidos y temas del eje de física para el currículum de educación media o secundaria. Estos contenidos conceptuales son necesarios para el desenvolvimiento ciudadano, en el sentido de enculturación científica, en la sociedad postmoderna (globalizada, con uso extendido de las tecnologías de información y comunicaciones, de la automatización y la informática, etc.). Posteriormente, en la sección 3, se contrasta dicha propuesta con el currículum de la educación secundaria, en cuanto a los contenidos de física, en Chile, España, México y Colombia, antes de mostrar las conclusiones, en la última sección.

\section{Episteme de la Física}

La reflexión conceptual sobre el mundo corpóreo, inanimado y mensurable, que nos rodea tuvo su origen en la filosofía natural que, grosso modo, nace con las especulaciones de la Grecia clásica en torno a la naturaleza y a las regularidades observadas en ella, principalmente en el movimiento de los astros. Por ello, la primera obra con el término physica (naturaleza) se debe a Aristóteles (s. IV a.c.) (Aristóteles, 1995). Así la idealización racional, causal y la conceptualización, se introducen como un primer elemento fundamental para la construcción del conocimiento acerca de la naturaleza. El segundo elemento que caracteriza a las ciencias naturales 
modernas, la contrastación y los procedimientos empíricos inductivos (observación, medición, experimentación e inferencia) se desarrollaron posteriormente y alcanzaron su máxima expresión en el siglo XVI, entre otros muchos, con las paradigmáticas contribuciones de Bacón, Kepler y Galileo. Si bien la escuela Alejandrina les antecediera, históricamente, por mucho. La filosofía natural del siglo XVI incorporaba entonces los procedimientos empíricos de contrastación con la realidad (fenomenología) a la reflexión racional del mundo (conceptualización), que se sistematiza a través de definiciones operativas por medio de construcciones matemáticas (metalenguaje) que describen sucintamente los procesos naturales. Por ejemplo, la conocida frase de Galileo (1638) "el gran libro de la naturaleza está escrito en símbolos matemáticos” y la magna obra de física, de Isaac Newton que se titula "Principios Matemáticos de la Filosofía Natural" (Newton, 1687).

De modo que, hasta comienzos del siglo XIX, quienes se dedicaban al estudio de las ciencias naturales se denominaban a sí mismos filósofos naturales. El calificativo de científicos lo introduce William Whewell (1847) para quien el método científico se sustenta en la "confluencia de inducciones", y el avance del conocimiento científico depende de la adecuación progresiva entre los hechos y las ideas que los ponen en conexión. Whewell (1840) se adelanta al concebir adecuadamente a la ciencia como un proceso creador y creativo, donde las ideas explican los hechos, y los hechos delimitan las ideas por medio de la contrastación, en una dialéctica faliblemente humana. En efecto, concebir el "método científico" como un procedimiento a seguir para inquirir la naturaleza, como si fuera un conjunto de pasos o etapas, es un reduccionismo de muy dudosa certidumbre, basado en una mitificación irracional del quehacer científico (Falcón, 2012).

La física estudia los cambios en los procesos naturales y para ello describe los entes que componen el mundo natural (materia y radiación), estudia los fenómenos a través de las características observables o cuantificables (magnitudes) y las interacciones entre los constituyentes. Las magnitudes físicas son algo más que cantidades, expresan cualidades o propiedades, por lo que contienen, 
además de números, otras ideas asociadas (en el sentido de la esencia Kantiana de noumeno) para estudiar las "apariencias" (phenomenun) de la naturaleza (physica) (Kant, 1989).

Toda descripción física de la naturaleza, como el conocimiento mismo, es reduccionista, en el sentido que no se trata de "copiar" los fenómenos y entender la totalidad del sistema en estudio, sino de describir cuáles características son las vinculantes, las relevantes, las causas que generan y describen los cambios en un sistema; en una porción seleccionada a ex profeso del universo sensible.

Para describir los cambios (fenómenos), se construyen en física conceptos genéricos, tanto reales como ideales, que se cuantifican operacionalmente por medio de magnitudes como: tiempo, distancia, fuerza, campo, energía, masa, carga eléctrica, etc. Las magnitudes expresan características, no siempre mensurables (la energía por ejemplo no se puede medir directamente con instrumento alguno), otras mensurables (masa, tiempo), colectivas o extensivas (como temperatura, densidad, etc.) y otras "individuales" o propias del objeto (masa, longitud, etc.) y también magnitudes direccionales o que dependen de la ubicación espacial relativa (como fuerza, velocidad, aceleración, campo, etc.). Tales conceptos se vinculan entre sí por medio de reglas o normas, prescritos por las teorías o modelos, ideados para describir la evolución de los procesos físicos y dar cuenta de la realidad observable o sensible. Puede entonces decirse que una teoría física es sólo un algebra involutiva de magnitudes observables (Hilbert y Ackermann, 1968), cuya prescripción se contrasta para delimitar la validez de su certidumbre, y permite deducir idealmente las "reglas" que regulan los fenómenos naturales, permitiendo en muchos casos su uso para la concreción de tecnologías.

Los elementos comunes de la episteme de las teorías físicas pueden resumirse en una tétrada de elementos característicos (Courant y Hilbert, 1937), a saber:

(i) Referentes espacio-temporales: es la delimitación de la parcela o porción del universo a estudiar, esto es el sistema o ensamble, o bien en establecer el marco referencial y las condiciones de frontera e iniciales.

(ii) Las especificaciones de los constituyentes: establece las 
propiedades colectivas y/o del medio que conforman la porción del universo (referente) de la teoría. Fundamentalmente establece las diferencias intrínsecas de los constituyentes o sus propiedades (por ejemplo: spin, conductividad eléctrica, grados de libertad, etc.).

(iii) Los observables: conjunto de magnitudes físicas que pueden obtenerse bien por su medición mediante dispositivos adecuados (ejemplo: temperatura, fuerza, voltaje, etc.) o bien, que puedan ser calculadas mediante reglas específicas, a partir de otras magnitudes (por ejemplo: calor, energía, entropía, etc.).

(iv) Las interacciones: conjunto de reglas (dinámica) que posibilita interconectar las componentes de la porción de universo estudiada. Los conceptos de campo (fuerza) y potencial (energía) que son comunes a toda descripción física de la naturaleza están vinculados a las interacciones y posibilitan encontrar las simetrías e invariancias por medio de las llamadas leyes de conservación o Teorema de Neather, (Landau, 1991).

Ahora bien, un í en física debería poseer también, a nivel macro, estos elementos dentro del diseño curricular (Manzanilla y Falcón, 2013, 2014). La inferencia entre los elementos de la epistemología de la física y el diseño curricular puede parecer artificial; sin embargo, algunos autores han señalado su importancia para la construcción de una "pedagogía científica", como una epistemología dialógica y crítica, tanto para la ciencia como para la educación (Bachelard, 1987, Garnica, 2017; Valenzuela, 2004). La aplicación de estas estructuras de conocimientos al diseño curricular, permite organizar los contenidos de física en el diseño curricular, como se aprecia en la tabla 1. Se advierte que la lista presentada es a título ilustrativo solamente, para orientar la clasificación de los contenidos y no es limitativa.

La secuencia de contenidos de la tabla 1 se elabora a partir de los constructos y magnitudes, desde los fundamentales o primigenios hasta los derivados o compuestos. La explicación de la secuencia para los no especialistas en física está fuera del alcance de este artículo, pero a modo de ejemplo basta decir que el concepto de velocidad es previo al de aceleración y éste al de fuerza. Que la dinámica 
de fluidos requiere de estos conceptos y así sucesivamente para las demás filas de la tabla 1, formando una concatenación lineal de arriba abajo, donde los nuevos constructos y magnitudes se construyen utilizando los anteriores.

Tabla 1. Contenidos generales de la física

\begin{tabular}{|c|c|c|c|}
\hline $\begin{array}{l}\text { (i) Referentes } \\
\text { Temas }\end{array}$ & $\begin{array}{c}\text { (ii) Contenidos } \\
\text { Fenómenos/Conceptos }\end{array}$ & $\begin{array}{l}\text { (iii) Observables } \\
\text { Magnitudes }\end{array}$ & $\begin{array}{c}\text { (iv) Reglas } \\
\text { Leyes-Modelos }\end{array}$ \\
\hline Cinemática & $\begin{array}{l}\text { Partícula reposo } \\
\text { Sistema de referencia } \\
\text { Movimiento rectilíneo uniforme } \\
\text { Caída libre } \\
\text { Movimiento unif. acelerado } \\
\text { Aceleración de gravedad } \\
\text { Mov. parabólico de proyectiles } \\
\text { Mov. circular uniforme }\end{array}$ & $\begin{array}{l}\text { Posición } \\
\text { Tiempo } \\
\text { Rapidez } \\
\text { Velocidad } \\
\text { Aceleración } \\
\text { Velocidad angular } \\
\text { Frecuencia Periodo }\end{array}$ & $\begin{array}{l}\cdot \text { Inercia } \\
\text { - Ecuación de } \\
\text { movimiento }\end{array}$ \\
\hline $\begin{array}{l}\text { Dinámica de la } \\
\text { partícula }\end{array}$ & $\begin{array}{l}\text { Reposo (inercia) } \\
\text { Equilibrio (traslación) } \\
\text { Fuerza resultante (neta) } \\
\text { "Fuerzas" de contacto, tensión } \\
\text { normal de un resorte y de } \\
\text { fricción } \\
\text { Movimiento circular no } \\
\text { uniforme } \\
\text { Aceleración centrípeta } \\
\text { Fuerzas resistivas }\end{array}$ & $\begin{array}{l}\text { Masa } \\
\text { Aceleración } \\
\text { Fuerza }\end{array}$ & $\begin{array}{l}\text { - 1ra Ley de Newton } \\
\text { - 2da Ley de Newton } \\
\text { - 3ra Ley de Newton } \\
\text { - Ley de Hooke }\end{array}$ \\
\hline $\begin{array}{l}\text { Trabajo, } \\
\text { energía y } \\
\text { momentum }\end{array}$ & $\begin{array}{l}\text { Impulso } \\
\text { Cantidad de movimiento } \\
\text { Colisiones elásticas e inelásticas } \\
\text { Coeficiente de restitución } \\
\text { Centro de masas y de gravedad } \\
\text { Movimiento de un sistema de } \\
\text { partículas } \\
\text { Sistemas de masa variable }\end{array}$ & $\begin{array}{l}\text { Trabajo } \\
\text { Energía } \\
\text { Potencia } \\
\text { Momentum lineal }\end{array}$ & $\begin{array}{l}\text { - Ley de conserva- } \\
\text { ción de la energía } \\
\text { (Helmholtz) } \\
\text { - Ley de conserva- } \\
\text { ción del momentum } \\
\text { lineal }\end{array}$ \\
\hline Gravitación & $\begin{array}{l}\text { Aceleración en caída libre } \\
\text { Campo gravitacional } \\
\text { Mareas } \\
\text { Sistema solar } \\
\text { Movimiento planetario y de } \\
\text { satélites }\end{array}$ & $\begin{array}{l}\text { Fuerza } \\
\text { Masa } \\
\text { Periodo } \\
\text { Potencial Gravita- } \\
\text { cional }\end{array}$ & $\begin{array}{l}\text { - Ley de Newton } \\
\text { de gravitación } \\
\text { universal } \\
\text { - Leyes de Kepler }\end{array}$ \\
\hline $\begin{array}{l}\text { Dinámica de } \\
\text { un sistema de } \\
\text { partículas }\end{array}$ & $\begin{array}{l}\text { Solido rígido } \\
\text { Cinemática rotacional } \\
\text { Traslación y rotación } \\
\text { Ejes y radios de giro } \\
\text { Energía cinética rotacional } \\
\text { Equilibrio rotacional } \\
\text { Giróscopos precesión }\end{array}$ & $\begin{array}{l}\text { Velocidad angular } \\
\text { Aceleración angular } \\
\text { Momento de Inercia } \\
\text { Momento de torsión } \\
\text { Momento angular } \\
\text { (cinético) }\end{array}$ & $\begin{array}{l}\text { - Ley de conserva- } \\
\text { ción del momentum } \\
\text { angular }\end{array}$ \\
\hline
\end{tabular}




\begin{tabular}{|c|c|c|c|}
\hline $\begin{array}{l}\text { Hidrostática y } \\
\text { fluidos }\end{array}$ & $\begin{array}{l}\text { Fluidos ideales } \\
\text { Presión atmosférica } \\
\text { Vaso comunicante } \\
\text { Flotación } \\
\text { Empuje } \\
\text { Osmosis capilaridad } \\
\text { Fluidos no newtonianos } \\
\text { Turbulencia }\end{array}$ & $\begin{array}{l}\text { Densidad } \\
\text { Presión } \\
\text { Caudal } \\
\text { Peso específico } \\
\text { Flujo másico } \\
\text { Tensión superficial } \\
\text { Viscosidad }\end{array}$ & $\begin{array}{l}\text { - Principio de Pascal } \\
\text { - Principio de Arquí- } \\
\text { medes } \\
\text { - Ecuación de conti- } \\
\text { nuidad } \\
\text { - Ley de Euler } \\
\text { - Ley de Bernoulli }\end{array}$ \\
\hline $\begin{array}{l}\text { Oscilaciones y } \\
\text { ondas mecánicas }\end{array}$ & $\begin{array}{l}\text { Movimiento oscilatorio } \\
\text { Péndulo } \\
\text { Movimiento armónico simple } \\
\text { Oscilaciones amortiguadas } \\
\text { Oscilaciones forzadas } \\
\text { Movimiento ondulatorio } \\
\text { Vibración y propagación de } \\
\text { perturbación } \\
\text { Onda longitudinal y transversal } \\
\text { Ondas progresivas } \\
\text { Ondas sinusoidales } \\
\text { Ondas sonoras } \\
\text { Reflexión y transmisión } \\
\text { Efecto Doppler } \\
\text { Ondas estacionarias } \\
\text { Interferencia } \\
\text { Resonancia }\end{array}$ & $\begin{array}{l}\text { Longitud de onda } \\
\text { Frecuencia } \\
\text { Velocidad de grupo } \\
\text { Velocidad de fase } \\
\text { Índice de refracción } \\
\text { Numero de Mach } \\
\text { Intensidad }\end{array}$ & $\begin{array}{l}\text { - Ecuación de onda } \\
\text { lineal } \\
\text { - Análisis de Fourier }\end{array}$ \\
\hline Termodinámica & $\begin{array}{l}\text { Calor } \\
\text { Dilatación térmica } \\
\text { Transferencia de calor } \\
\text { Conducción, convección, } \\
\text { Radiación } \\
\text { Emisión, absorción y reflexión } \\
\text { de energía radiante } \\
\text { Equivalente mecánico del calor } \\
\text { Efecto invernadero } \\
\text { Gases Ideales } \\
\text { Calor específico de un gas ideal } \\
\text { Procesos adiabáticos } \\
\text { Cambio de fase } \\
\text { Evaporación, condensación } \\
\text { Ebullición, fusión, congelación } \\
\text { Energía y cambios de fase } \\
\text { Cero absoluto } \\
\text { Energía interna } \\
\text { Proceso adiabático } \\
\text { Máquinas térmicas } \\
\text { Procesos reversibles e irrever- } \\
\text { sibles } \\
\text { Estados accesibles } \\
\text { Orden y caos }\end{array}$ & $\begin{array}{l}\text { Temperatura calor } \\
\text { específico } \\
\text { Cantidad de calor } \\
\text { Eficiencia térmica } \\
\text { Entalpia } \\
\text { Entropía } \\
\text { Energía libre }\end{array}$ & $\begin{array}{l}\cdot \text { Ley cero sobre } \\
\text { transferencia de } \\
\text { calor } \\
\text { - Ley de Newton } \\
\text { sobre enfriamiento } \\
\text { - Primera ley de la } \\
\text { termodinámica } \\
\text { - Teoría cinética de } \\
\text { los gases } \\
\text { - Ecuación de estado } \\
\text { gas ideal } \\
\text { - Ecuación Clausius- } \\
\text { Clapeyron } \\
\text { - Segunda ley de la } \\
\text { termodinámica }\end{array}$ \\
\hline Electricidad & $\begin{array}{l}\text { Cargas eléctricas } \\
\text { Electricidad estática } \\
\text { Inducción eléctrica } \\
\text { Líneas de campo eléctrico } \\
\text { Dipolo eléctrico } \\
\text { Movimiento de partículas } \\
\text { cargadas }\end{array}$ & $\begin{array}{l}\text { Carga eléctrica } \\
\text { Campo eléctrico } \\
\text { Flujo eléctrico } \\
\text { Potencial eléctrico } \\
\text { Corriente eléctrica } \\
\text { Capacidad eléctrica } \\
\text { Permitividad } \\
\text { eléctrica }\end{array}$ & $\begin{array}{l}\text { - Ley de conser- } \\
\text { vación de la carga } \\
\text { eléctrica } \\
\text { - Ley de Coulomb } \\
\text { - Ley de Gauss } \\
\text { - Modelo de conduc- } \\
\text { ción eléctrica } \\
\text { - Ley de Ohm }\end{array}$ \\
\hline
\end{tabular}




\begin{tabular}{|c|c|c|c|}
\hline & $\begin{array}{l}\text { Conductores en equilibrio } \\
\text { electrostático } \\
\text { Blindaje (jaula de Faraday) } \\
\text { Potencial y diferencia de } \\
\text { potencial } \\
\text { Capacitancia dieléctricos } \\
\text { Capacitores en serie y en } \\
\text { paralelo } \\
\text { Polarización eléctrica } \\
\text { Corriente y resistencia } \\
\text { Efecto de la temperatura } \\
\text { Semiconductores, supercon- } \\
\text { ductores } \\
\text { Circuitos de corriente directa } \\
\text { (DC) } \\
\text { Fuerza electromotriz } \\
\text { Resistores en serie y en paralelo } \\
\text { Circuitos RC }\end{array}$ & $\begin{array}{l}\text { Intensidad de cam- } \\
\text { po eléctrico } \\
\text { Vector de desplaza- } \\
\text { miento eléctrico } \\
\text { Resistividad } \\
\text { Resistencia } \\
\text { Densidad de co- } \\
\text { rriente } \\
\text { Potencia eléctrica }\end{array}$ & $\begin{array}{l}\text { - Efecto Joule } \\
\text { - Leyes de Kirchhoff }\end{array}$ \\
\hline Magnetismo & $\begin{array}{l}\text { Campos y fuerzas magnéticas } \\
\text { Movimiento de cargas en campo } \\
\text { magnético uniforme } \\
\text { Fuerza magnética sobre una } \\
\text { corriente } \\
\text { Momento de torsión sobre una } \\
\text { espira } \\
\text { Fuerza magnética entre dos } \\
\text { conductores } \\
\text { Solenoide } \\
\text { Materiales ferromagnéticos, dia- } \\
\text { magnéticos y paramagnéticos } \\
\text { Histéresis } \\
\text { Campo magnético terrestre } \\
\text { Auroras boreales }\end{array}$ & $\begin{array}{l}\text { Campo magnético } \\
\text { Susceptibilidad } \\
\text { magnética } \\
\text { Flujo magnético } \\
\text { Inducción mag- } \\
\text { nética } \\
\text { Magnetización } \\
\text { Intensidad de cam- } \\
\text { po magnético }\end{array}$ & $\begin{array}{l}\text { - Ley de Gauss para } \\
\text { el magnetismo } \\
\text { - El efecto Hall } \\
\text { - Ley de Biot-Savart } \\
\text { - Ley de Ampère }\end{array}$ \\
\hline $\begin{array}{l}\text { Electromagne- } \\
\text { tismo }\end{array}$ & $\begin{array}{l}\text { Leyes de inducción de Faraday } \\
\text { Fem inducida y campos eléc- } \\
\text { tricos } \\
\text { Generadores y motores } \\
\text { Autoinducción, inductancia } \\
\text { Inductancia mutua } \\
\text { Corrientes de Eddy } \\
\text { Energía en un campo magnético } \\
\text { Circuitos RL y LC } \\
\text { Oscilaciones en un circuito LC } \\
\text { Circuito RLC } \\
\text { Circuitos de corriente alterna } \\
\text { AC } \\
\text { Potencia y factor potencia } \\
\text { Resonancia } \\
\text { Transformadores, rectificadores } \\
\text { y filtros } \\
\text { Ondas electromagnéticas } \\
\text { Corriente de desplazamiento } \\
\text { Ondas electromagnéticas planas } \\
\text { Energía de ondas electromag- } \\
\text { néticas } \\
\text { Presión de radiación } \\
\text { Dipolo y antenas } \\
\text { Espectro electromagnético }\end{array}$ & $\begin{array}{l}\text { Vector de Poynting } \\
\text { Frecuencia de } \\
\text { resonancia } \\
\text { Factor potencia } \\
\text { Voltaje y corriente } \\
\text { eficaz } \\
\text { Valor DC de una } \\
\text { señal alterna }\end{array}$ & $\begin{array}{l}\text { - Ley de Faraday } \\
\text { - Ley de Lenz } \\
\text { - Ley de Ampère - } \\
\text { Maxwell } \\
\text { - Ecuaciones de } \\
\text { Maxwell }\end{array}$ \\
\hline
\end{tabular}




\begin{tabular}{|c|c|c|c|}
\hline Óptica & $\begin{array}{l}\text { Naturaleza de la luz } \\
\text { Color } \\
\text { Rayos paraxiales y frentes de } \\
\text { onda } \\
\text { Reflexión y refracción } \\
\text { Reflexión total interna } \\
\text { Dispersión } \\
\text { Formación de las imágenes } \\
\text { Espejos planos y esféricos } \\
\text { Lentes delgadas } \\
\text { Aberraciones e instrumentos } \\
\text { ópticos } \\
\text { Interferencia } \\
\text { Experimento de doble ranura } \\
\text { de Young } \\
\text { Cambio de fase debido a } \\
\text { reflexión } \\
\text { Interferómetro de Michelson } \\
\text { Polarización ángulo de Brewster } \\
\text { Rejilla de difracción }\end{array}$ & $\begin{array}{l}\text { Índice de refracción } \\
\text { Distancia focal } \\
\text { Longitud de onda } \\
\text { Frecuencia } \\
\text { Velocidad de propa- } \\
\text { gación } \\
\text { Intensidad } \\
\text { Longitud de trayec- } \\
\text { toria óptica (LTO) }\end{array}$ & $\begin{array}{l}\text { - Modelo de } \\
\text { Maxwell de la Luz } \\
\text { - Ley de reflexión } \\
\text { - Ley de Snell } \\
\text { - Ecuación de las } \\
\text { lentes delgadas } \\
\text { - Principio de } \\
\text { Huygens } \\
\text { - Ley de Bragg } \\
\text { - Difracción de Fres- } \\
\text { nel y de Fraunhofer } \\
\text { - Ley de Malus }\end{array}$ \\
\hline $\begin{array}{l}\text { Física atómica } \\
\text { y mecánica } \\
\text { cuántica }\end{array}$ & $\begin{array}{l}\text { Experimento de Michelson- } \\
\text { Morley } \\
\text { Principio de relatividad de } \\
\text { Einstein } \\
\text { Consecuencias de la relatividad } \\
\text { Momento lineal y energía } \\
\text { relativista } \\
\text { Equivalencia masa y energía } \\
\text { Radiación de cuerpo negro } \\
\text { Fotones y ondas } \\
\text { Experimento de Young con } \\
\text { fotones y electrones } \\
\text { Espectros atómicos de los gases } \\
\text { de emisión y de absorción } \\
\text { Modelos de Thompson y Ru- } \\
\text { therford } \\
\text { Modelo de Bohr del átomo de } \\
\text { hidrógeno } \\
\text { Dualidad onda partícula } \\
\text { Mecánica cuántica } \\
\text { Partícula en un pozo de po- } \\
\text { tencial } \\
\text { Aplicaciones del efecto túnel } \\
\text { Oscilador armónico en mecáni- } \\
\text { ca cuántica } \\
\text { Funciones de onda para el } \\
\text { hidrógeno } \\
\text { Tabla periódica } \\
\text { Transiciones electrónicas, es- } \\
\text { pontáneas y estimuladas (láser) }\end{array}$ & $\begin{array}{l}\text { Masa en reposo } \\
\text { Longitud de Onda } \\
\text { Compton } \\
\text { Longitud de Onda } \\
\text { de De Boglie } \\
\text { Números Cuánticos } \\
\text { Temperatura } \\
\text { efectiva }\end{array}$ & $\begin{array}{l}\text { - Teoría de la Rela- } \\
\text { tividad Especial de } \\
\text { Einstein } \\
\text { - Ecuaciones de } \\
\text { Lorentz } \\
\text { - Hipótesis de } \\
\text { Planck } \\
\text { - Ley de desplaza- } \\
\text { miento de Wiens } \\
\text { - Ley de Stefan- } \\
\text { Boltzmann } \\
\text { - Efecto fotoeléc- } \\
\text { trico } \\
\text { - Modelo de Bohr } \\
\text { - Efecto Compton } \\
\text { - Hipotesis de Boglie } \\
\text { - Principio de } \\
\text { incertidumbre de } \\
\text { Heisemberg } \\
\text { - Ecuación de } \\
\text { Schrödinger } \\
\text { - Efecto túnel }\end{array}$ \\
\hline Física nuclear & $\begin{array}{l}\text { Descubrimiento de la Radiac- } \\
\text { tividad } \\
\text { Modelos nucleares } \\
\text { propiedades de los núcleos } \\
\text { Energía de enlace nuclear } \\
\text { Radiación alfa, beta y gama } \\
\text { Isotopos e isóbaros }\end{array}$ & $\begin{array}{l}\text { Numero atómico } \\
\text { Peso atómico } \\
\text { Vida media } \\
\text { Energía de enlace } \\
\text { Actividad radiactiva } \\
\text { Dosis absorbida }\end{array}$ & $\begin{array}{l}\cdot \text { Ley de decaimien- } \\
\text { to radiactivo } \\
\text { - Ley de Moseley } \\
\text { - Ley de conser- } \\
\text { vación número } \\
\text { bariónico } \\
\text { - Teorías de Gran } \\
\text { Unificación (GTU) }\end{array}$ \\
\hline
\end{tabular}


Radiactividad natural y series radiactivas

Reacciones nucleares

Resonancia magnética nuclear

Fisión y fusión nuclear

Reacción en cadena

Reactores nucleares

Protección radiológica y dosi-

metría

Fuerzas fundamentales en la

naturaleza

Clasificación de partículas su-

batómicas: hadrones, bariónes, leptones, quarks, etc.

Los referentes de la tabla 1 pueden renombrarse en términos fenoménicos de una forma más comprensible para quien no es especialista en física, o para los propios educandos, evocando los fenómenos usuales de la naturaleza; sin pérdida alguna de generalidad. Nótese que los temas nombrados en las primeras tres filas de la tabla 1 se refieren al movimiento de los cuerpos en la naturaleza, por lo tanto, la organización interna y conceptual de la física podemos expresarla sucintamente como en la tabla 2.

Tabla 2. Contenidos fenoménicos resumidos de la física

\begin{tabular}{|c|c|c|c|}
\hline $\begin{array}{l}\text { (i) Referentes } \\
\text { Fenómenos } \\
\text { asociados }\end{array}$ & $\begin{array}{l}\text { (ii) Contenidos } \\
\text { Conceptos, } \\
\text { situaciones y } \\
\text { aplicaciones }\end{array}$ & $\begin{array}{l}\text { (iii) Observables } \\
\text { Magnitudes } \\
\text { Características }\end{array}$ & $\begin{array}{l}\text { (iv) Reglas } \\
\text { Leyes-Modelos } \\
\text { Fundamentales }\end{array}$ \\
\hline $\begin{array}{l}\text { 1. Movimiento de } \\
\text { traslación }\end{array}$ & $\begin{array}{l}\text { Cinemática y } \\
\text { dinámica de la } \\
\text { partícula }\end{array}$ & $\begin{array}{l}\text { Posición, tiempo } \\
\text { Velocidad } \\
\text { Aceleración } \\
\text { Masa } \\
\text { Fuerza } \\
\text { Energía } \\
\text { Momentum lineal }\end{array}$ & $\begin{array}{l}\text { - Ecuación de } \\
\text { movimiento } \\
\text { - Leyes de Newton } \\
\text { - Conservación de la } \\
\text { energía } \\
\text { - Conservación del } \\
\text { momentum lineal }\end{array}$ \\
\hline $\begin{array}{l}\text { 2. Movimiento } \\
\text { rotacional y la } \\
\text { gravitación }\end{array}$ & $\begin{array}{l}\text { Cinemática y } \\
\text { dinámica del sólido } \\
\text { gravitación }\end{array}$ & $\begin{array}{l}\text { Potencial gravita- } \\
\text { cional } \\
\text { Momento de inercia } \\
\text { Momento de torsión } \\
\text { Momento angular } \\
\text { (cinético) }\end{array}$ & $\begin{array}{l}\text { - Ley de Gravitación } \\
\text { Universal } \\
\text { - Leyes de Kepler } \\
\text { - Conservación del } \\
\text { momentum angular }\end{array}$ \\
\hline
\end{tabular}




\begin{tabular}{|c|c|c|c|}
\hline $\begin{array}{l}\text { 3. Movimiento de } \\
\text { los fluidos }\end{array}$ & $\begin{array}{l}\text { Mecánica de los } \\
\text { fluidos }\end{array}$ & $\begin{array}{l}\text { Densidad } \\
\text { Presión } \\
\text { Caudal } \\
\text { Viscosidad }\end{array}$ & $\begin{array}{l}\text { - Principio de } \\
\text { Arquímedes } \\
\text { - Ecuación de } \\
\text { continuidad } \\
\text { - Ley de Euler } \\
\text { - Ley de Bernoulli }\end{array}$ \\
\hline $\begin{array}{l}\text { 4. Naturaleza del } \\
\text { sonido }\end{array}$ & $\begin{array}{l}\text { Oscilaciones y } \\
\text { acústica }\end{array}$ & $\begin{array}{l}\text { Longitud de Onda } \\
\text { Frecuencia } \\
\text { Índice de Refracción } \\
\text { Numero de Mach } \\
\text { Intensidad }\end{array}$ & $\begin{array}{l}\text { - Ecuación de onda } \\
\text { lineal }\end{array}$ \\
\hline $\begin{array}{l}\text { 5. Naturaleza del } \\
\text { calor }\end{array}$ & Termodinámica & $\begin{array}{l}\text { Temperatura } \\
\text { Calor específico } \\
\text { Cantidad de calor } \\
\text { Entalpia } \\
\text { Entropía }\end{array}$ & $\begin{array}{l}\text { - 1ra y } 2 \text { da Ley de la } \\
\text { termodinámica } \\
\text { - Ley de Newton } \\
\text { sobre enfriamiento } \\
\text { - Ecuación del gas } \\
\text { ideal }\end{array}$ \\
\hline $\begin{array}{l}\text { 6. Naturaleza de la } \\
\text { electricidad }\end{array}$ & $\begin{array}{l}\text { Electrostática y } \\
\text { electrocinética }\end{array}$ & $\begin{array}{l}\text { Carga eléctrica } \\
\text { Campo eléctrico } \\
\text { Potencial eléctrico } \\
\text { Corriente eléctrica }\end{array}$ & $\begin{array}{l}\text { - Conservación de la } \\
\text { carga eléctrica } \\
\text { - Ley de Coulomb } \\
\text { - Ley de Ohm } \\
\text { - Efecto Joule } \\
\text { - "Leyes" de } \\
\text { Kirchhoff }\end{array}$ \\
\hline $\begin{array}{l}\text { 7. Naturaleza del } \\
\text { magnetismo }\end{array}$ & Electromagnetismo & $\begin{array}{l}\text { Campo magnético } \\
\text { Flujo magnético } \\
\text { Inducción magné- } \\
\text { tica } \\
\text { Vector de Poynting }\end{array}$ & $\begin{array}{l}\text { - Ecuaciones de } \\
\text { Maxwell }\end{array}$ \\
\hline $\begin{array}{l}\text { 8. Naturaleza de } \\
\text { la luz }\end{array}$ & $\begin{array}{l}\text { Óptica geométrica } \\
\text { y óptica física }\end{array}$ & $\begin{array}{l}\text { Índice de refracción } \\
\text { Intensidad } \\
\text { Longitud de trayec- } \\
\text { toria óptica (LTO) }\end{array}$ & $\begin{array}{l}\text { - Leyes de la óptica } \\
\text { geométrica } \\
\text { - Principio de } \\
\text { Huygens }\end{array}$ \\
\hline $\begin{array}{l}\text { 9. Naturaleza de la } \\
\text { radioactividad }\end{array}$ & $\begin{array}{l}\text { Física atómica, } \\
\text { física nuclear, me- } \\
\text { cánica cuántica }\end{array}$ & $\begin{array}{l}\text { Masa en reposo } \\
\text { Números cuánticos } \\
\text { Numero atómico } \\
\text { Peso atómico } \\
\text { Vida media } \\
\text { Energía de enlace } \\
\text { Actividad radiactiva }\end{array}$ & $\begin{array}{l}\text { - Teoría especial de } \\
\text { la relatividad } \\
\text { - Modelo de Bohr } \\
\text { - Principio de } \\
\text { incertidumbre de } \\
\text { Heisenberg } \\
\text { - Ecuación de } \\
\text { Schrödinger } \\
\text { - Teorías de Gran } \\
\text { Unificación (GTU) }\end{array}$ \\
\hline
\end{tabular}


En la sociedad postmoderna, los fenómenos de la propagación de ondas electromagnéticas, tecnologías satelitales, radiactividad y física atómica, entre otros, adquieren particular importancia, tanto por sus alcances, como por las más modernas aplicaciones (laser, TIC, etc.); sin embargo, en el currículum de educación media estos temas son soslayados. En la siguiente sección se emplean las tablas 1 y 2 para diagnosticar la completitud, alcance y secuencia de los contenidos de física en la educación media.

\section{Diagnosis curricular de la física en educación media}

Para indagar la implementación curricular de los contenidos en el eje de física, se procede a comparar las mallas curriculares del 1ro a 4to año de la educación media de Chile con las correspondientes de Colombia, México y España. La comparación con los países hispanohablantes está justificada por razones metodológicas y fundamentales. En primer término, una comparación de contenidos en física para el área particular del conocimiento en ciencias naturales, supone comparar con sistemas educativos similares (Ruiz y Schoo, 2014 Rivas y Sánchez, 2016, Cox 2001) en perfiles, alcances y valores para la educación media (secundaria o de bachillerato según las diferentes denominaciones). La tabla 3, muestra las generalidades de la organización por grados o niveles de estos currículums.

Se observa en la muestra estudiada, que en todos los casos se considera una escolaridad desde los 6-7 años para el inicio de la primaria; 11-13 para la segunda etapa (llámese secundaria o media) y culmina con un ciclo pre-universitario o laboral hacia los 17-18 años. Si bien en cada país la escolaridad obligatoria varía desde los 9 grados (México) hasta los 12 grados (Chile). Es común también considerar los últimos dos años (Chile, Colombia España) o tres años (México) como el ciclo final de currículum electivo, en el cual existen múltiples opciones (desde sólo tres como el bachillerato en España: Ciencias, artes o humanidades; hasta más de una decena en el resto de los otros casos de la muestra). 
Tabla 3. Niveles de escolaridad por país

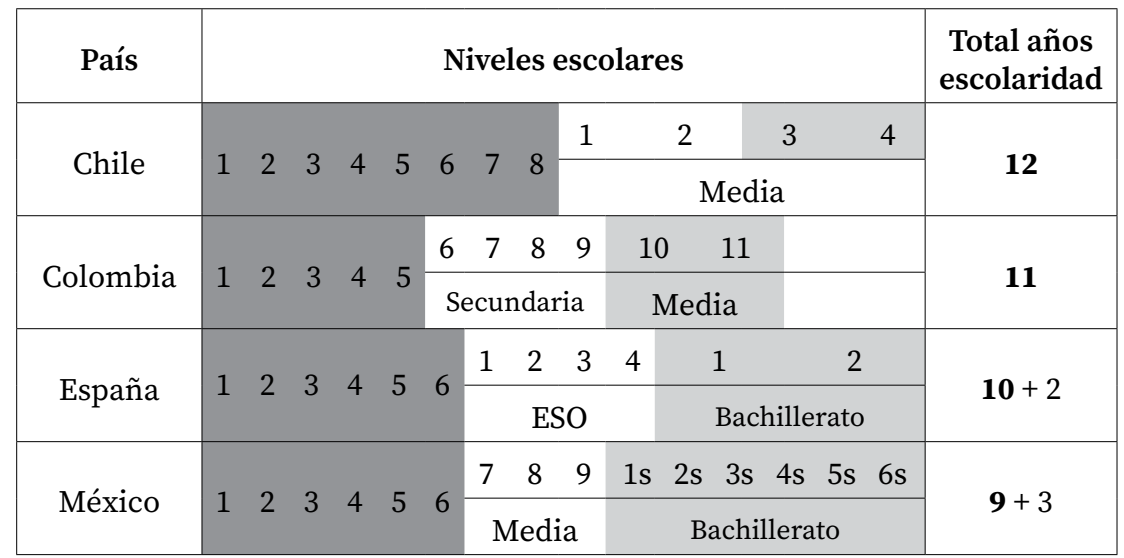

Nota: El sombreado oscuro denota la educación primaria, el sombreado claro la educación diversificada. En la última columna las negrillas destacan los años de escolaridad obligatoria. La letra "s" indica escolaridad semestral. Fuente: Elaboración propia.

Un desglose de la ubicación de los cursos con contenidos específicos de física se presenta en la tabla 4, con la finalidad de comparar la instrucción en cultura científica, particularmente en física, a nivel de la escolaridad entre los 11-13 años y los 17-18 años que, dependiendo de la denominación autóctona, se considera enseñanza media, secundaria y/o bachillerato.

Nótese que los contenidos de física propiamente dichos, se suelen colocar al final del ciclo de escolaridad en todos los casos estudiados, vale decir en el ciclo diversificado, casi como una especialización temprana para quienes aspiran a seguir estudios superiores en carreras de tipo técnico-científicas. Los perfiles de la escolaridad obligatoria (Braslavsky, 2001) pregonan la formación ciudadana en la cultura científica y con conocimientos de los fenómenos de la naturaleza y del ambiente, con interés en los métodos, logros y alcances de la ciencia y la tecnología, pero en los currículums implementados soslayan la enseñanza formal de la naturaleza (física) a solo una porción de quienes pretendan especializarse en esas áreas y no para todos los ciudadanos. 
Tabla 4. Niveles de escolaridad en física por país

\begin{tabular}{|c|c|c|c|c|c|c|c|c|}
\hline País & \multicolumn{7}{|c|}{ Niveles escolares } & $\begin{array}{l}\text { Total años } \\
\text { Cursos de }\end{array}$ \\
\hline \multirow{2}{*}{ Chile } & \multirow{2}{*}{$6^{\circ}$} & \multirow{2}{*}{$7^{\circ}$} & \multirow{2}{*}{$8^{\circ}$} & $1 \mathrm{CN}$ & $2 \mathrm{CN}$ & $3 \mathrm{~F}$ & $4 \mathrm{~F}$ & \multirow{2}{*}{$2+2$} \\
\hline & & & & \multicolumn{4}{|c|}{ Media } & \\
\hline \multirow{2}{*}{ Colombia } & $6^{\circ} \mathrm{CN}$ & $7^{\circ} \mathrm{CN}$ & $8^{\circ} \mathrm{CN}$ & $9^{\circ} \mathrm{CN}$ & $10^{\circ} \mathrm{F}$ & $11^{\circ} \mathrm{F}$ & & \multirow{2}{*}{$4+2$} \\
\hline & \multicolumn{3}{|c|}{ Secundaria } & \multicolumn{3}{|c|}{ Media } & & \\
\hline \multirow{2}{*}{ España } & \multirow{2}{*}{$6^{\circ}$} & 1 & 2 FyQ & 3 FyQ & 4 FyQ & 1 FyQ & $1 \mathrm{~F}$ & \multirow{2}{*}{$2+3$} \\
\hline & & \multicolumn{3}{|c|}{ ESO } & \multicolumn{3}{|c|}{ Bachillerato } & \\
\hline \multirow{2}{*}{ México } & \multirow{2}{*}{$6^{\circ}$} & 7 & $8 \mathrm{~F}$ & 9 & $1 \mathrm{~s} 2 \mathrm{~s} 3$ & $\mathrm{sF} 4 \mathrm{sF} 5$ & $s F \quad 6 s F$ & \multirow{2}{*}{$1+2$} \\
\hline & & \multicolumn{3}{|c|}{ Media } & \multicolumn{3}{|c|}{ Bachillerato } & \\
\hline
\end{tabular}

Nota: El sombreado oscuro denota la educación primaria, el sombreado claro la educación diversificada. En la última columna las negrillas destacan los años de escolaridad obligatoria. La letra "s" indica escolaridad semestral, FyQ la asignatura "física y química”, F para las asignaturas de "física” y CN para las denominadas ciencias naturales. Fuente: Elaboración propia.

Y entonces hay quienes se asombran de por qué, por ejemplo, un ciudadano común, aún siendo un profesional de carrera no técnica, desconoce conceptos elementales de gran utilidad diaria como: que la corriente es diferente al voltaje; el significado del valor en vatios de una lámpara; o la diferencia que existe entre voltaje alterno y continuo; o que la temperatura no se transmite; o que vivimos rodeados de materiales radiactivos y eso no significa necesariamente un peligro para la salud; o que una antena receptora de telefonía o un cable de canalización eléctrica cerca de nuestras casas no necesariamente entraña peligro para los humanos; o la diferencia entre radiación infrarroja y ultravioleta, etc.

En todos los casos de la muestra se dedican contenidos de física tempranamente y en forma obligatoria, después de la enseñanza primaria, unas veces con la denominación específica de física (México en $2^{\circ}$ medio) otras como ciencias naturales: $1^{\circ}$ y $2^{\circ}$ medio de Chile; y $6^{\circ}$ y $7^{\circ}$ de la etapa secundaria en Colombia, o como física y química” 
en 2do y 3er año de la enseñanza secundaria obligatoria (ESO) de España. Si bien la intención es proveer una cultura científica y una cierta alfabetización científica de todos los ciudadanos, como se declara en el perfil del currículum prescrito; esto no se hace en el currículum implementado de los países estudiados. En efecto, al examinar los detalles de los contenidos, alcances y secuencias temáticas; se destaca que los temas de mayor aplicación tecnológica de nuestra cultura postmoderna (electricidad, magnetismo, naturaleza del calor y de la luz, radiactividad y física atómica-nuclear) quedan soslayados en la escolaridad obligatoria, como se muestra la infografía siguiente.

Figura 1. Infografía comparativa de contenidos y secuencias temáticas en física, en los currículums de educación secundaria
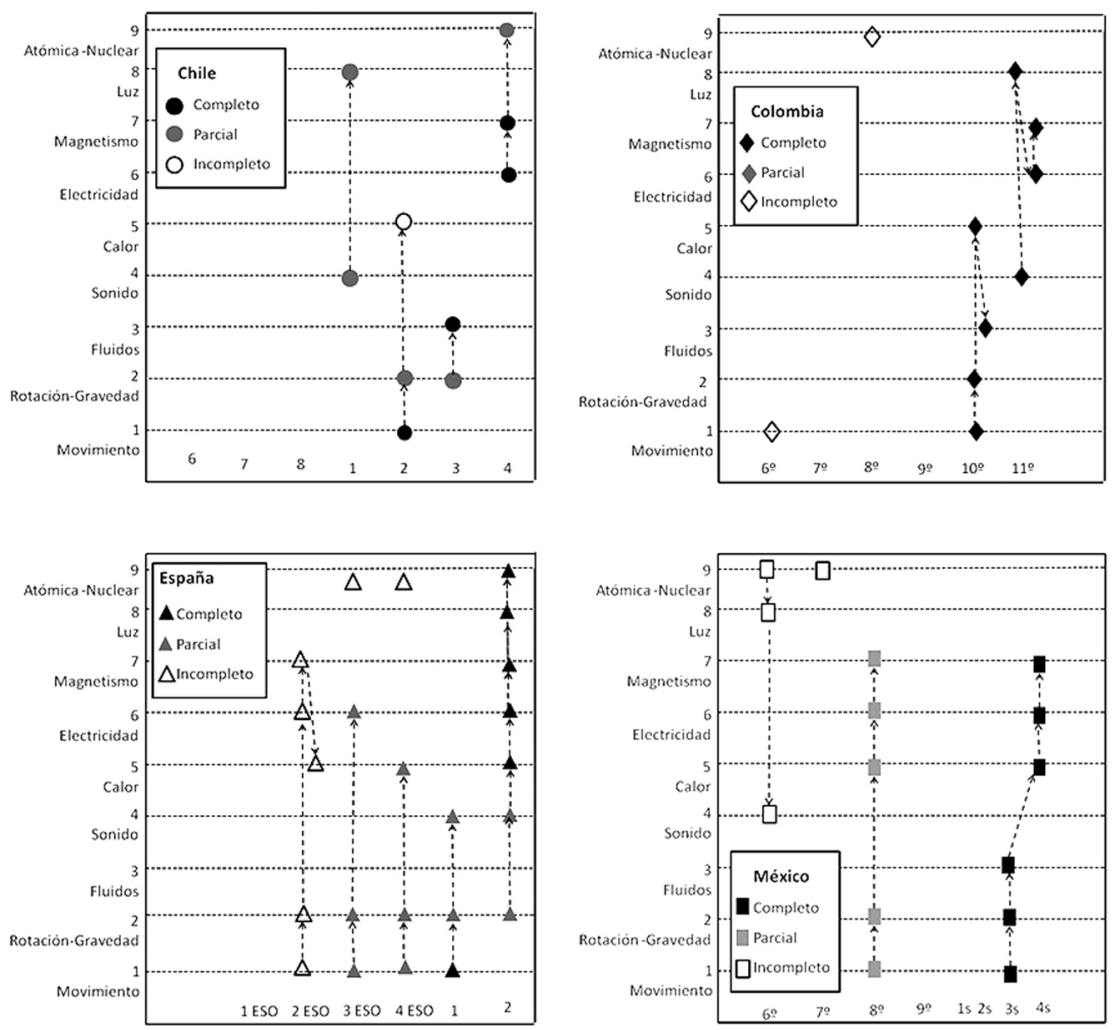

Fuente: Elaboración propia. 
Se observa una gran heterogeneidad en la distribución y alcance de los contenidos por grados de escolaridad en la muestra; también que la cobertura de los temas se alcanza sobre todo en los dos últimos años de la secuencia escolar (etapa diversificada). Las asignaturas de ciencias naturales y de física colocadas en los primeros años de la educación media y secundaria no cumplen su objetivo de proveer cultura científica en la escolaridad obligatoria porque no cubren los contenidos fenoménicos de la física. En Chile sólo dos temas parciales, dispersos y sin las herramientas mínimas de comprensión que les dé coherencia (sin cinemática, ni dinámica ni vectores ni trigonometría elemental, por ejemplo). En Colombia, en secundaria solo la tabla periódica; en España una repetición innecesaria y parcial en los primeros años de la ESO, dejando fuera los temas de luz, sonido y fluidos, que tampoco se abordan en la asignatura similar de México. Los tópicos relacionados a las nuevas tecnologías del siglo XXI: Ondas electromagnéticas (telefonía celular, radar, microondas, etc), fibras ópticas, láser, semiconductores y superconductividad, efecto fotoeléctrico (aprovechamiento de la energía solar), radiactividad y energía nuclear, resonancia magnética; etc. que requieren del tema 9, está ausente en casi todas las asignaturas y sólo al final de la escolaridad se imparte de soslayo en el último tramo, del último año del bachillerato o educación media. Por último, a excepción de México y de los dos años del bachillerato en España, la secuencia y selección de los contenidos no es lineal y no sigue el orden discursivo interno de la episteme de la física; quizá por ello la dificultad de desempeño de los estudiantes en la asignatura de física.

\section{Conclusiones y recomendaciones}

La pretensión de proveer una cultura científica en los estudiantes de la educación media, secundaria o de bachillerato, según la nomenclatura que se use para referirse a la formación escolar entre los 12 y 18 años, no se expresa en los contenidos programáticos y temarios de las asignaturas denominadas como ciencias naturales y física, 
por cuanto los temas que se abordan, en la muestra de países estudiados: Chile, Colombia España y México, no cubre los principales fenómenos y discursos sobre la naturaleza, donde temas importantes como las leyes que describen la naturaleza de la luz, del calor, de la electricidad del magnetismo, de la radiactividad, etc. están ausentes en dichas asignaturas. Por tanto, hay una gran disonancia entre el currículum escolar prescrito y el currículum implementado en el área de física a nivel de la escolaridad obligatoria.

Se sugiere la revisión de los contenidos, alcances y enfoques de esas asignaturas, desde un punto de vista fenomenológico y conceptual, soslayando el metalenguaje matemático en un primer nivel de comprensión para abarcar todos los tópicos de la ciencia física y de las tecnologías que se fundamentan en dichas leyes y modelos. Dicha asignatura debería ser de carácter obligatorio en los primeros años de la escolaridad media. Luego, como es usual, en el ciclo diversificado del último bienio, retomar la totalidad de esos temas de física, con un enfoque riguroso y con la incorporación del metalenguaje abstracto, pero cubriendo todas las grandes síntesis teóricas de la física del siglo XX.

Una inspección del alcance de los contenidos en física de la educación secundaria, revela que la selección de contenidos en física sigue siendo tradicional y con una selección de tópicos centrada en las teorías física del siglo XIX; y solo en algunos pocos casos, se le dedica alguna mención, casi de soslayo, a la física hasta comienzos del siglo XX; es decir con casi un siglo de atraso en cuanto al desarrollo efectivo de las ciencias naturales.

Por último, hay que resaltar que la heurística aquí desarrollada, de emplear la episteme de la física, para clasificar y ordenar sistémicamente los contenidos, puede emplearse como criterio no sólo para analizar los currículums, sino que podría ser una herramienta para la educación comparada y también para la acreditación hemisférica de los títulos de educación media. Con una heurística análoga, cada disciplina particular puede elaborar otros criterios diferentes, basado en la episteme propia de cada una, ordenando los constructos e ideas, los cuales han de tener, con mayor motivo, una concatenación y secuencia interna, 
bien sea para matemáticas, química, biología e incluso disciplinas humanistas como historia o idiomas.

\section{Referencias}

Arias, M., Navarro, M. (2017) Epistemología, Ciencia y Educación Científica: premisas, cuestionamientos y reflexiones para pensar la cultura científica. Revista E. Actualidades Investigativas en Educación 17,3, 774-794.

Aristóteles (1995) [s. IV A.C.] Physica. Traducción española de Echandía G. Barcelona: Editorial Gredos, S.A.

Ausubel, D.P., Novak, J.D. y Hanesian, H. (1983). Psicología Educativa: un punto de vista cognoscitivo. México: Ed.Trillas.

Bachelard, G. (1987) La formación del espiritu científico. México: Edit. Siglo XXI.

Braslavsky, C., Ed. (2001). La educación secundaria. ¿Cambio o inmutabilidad? Análisis y debate de procesos europeos y latinoamericanos contemporáneos. Buenos Aires: Santillana.

Chevallard, Y. (1998). La transposición didáctica: del saber sabio al saber enseñado. Buenos Aires: Aique Grupo Editorial.

Collins, A., Bielaczyc, K. (1999) The Enculturation of Educational Thinking. Journal of the Learning Sciences, 8, 129-138.

Courant, R.; Hilbert, D. (1937) Methoden der Mathematischen Physik, Berlin: Springer.

Cox, C. (2001). Políticas educacionales y procesos de cambio en la educación media de Chile en los años noventa. C. Braslavsky (Ed.). La educación secundaria. ¿Cambio o inmutabilidad? Análisis y debate de procesos europeos y latinoamericanos contemporáneos (pp. 283-336). Buenos Aires: Ed. Santillana.

Falcón, N. (2012) Mitos y Sofismas de la Cultura Cientifica en la Sociedad Postmoderna. Sapiens Research, 1, 18-20.

Fourez, G. (1996). Alphabétisation scientifique et technique. Essai sur les finalités de l'enseignement des sciences. Revue française de pédagogie Année, 115, 133-134.

Galileo, G (1981) [1638] Consideraciones y demostraciones matemáticas sobre dos nuevas ciencias. Madrid: Solis y Sádaba, Eds. 
Garnica, N. (2017) La epistemología histórica en perspectiva Bachelard en la epistemología francesa. Perspectivas Metodológicas, 20, 14-24.

Gil-Pérez, D.,Vilches-Peña, A. (2001). Una Alfabetización Científica para el Siglo XXI: Obstáculos y Propuestas de Actuación. Revista Investigación en la Escuela, 43, 27-37.

Gómez, J. (2012). Cultura: sus significados y diferentes modelos de cultura científica y técnica. Revista Iberoamericana de Educación, 58, 15-33.

Hilbert, D. Ackermann, W (1968) Elementos de lógica teórica. Madrid: Editorial: Tecnos

Kant, I. (1989) [1786] Principios metafísicos de la ciencia de la naturaleza. Madrid: Alianza Ed.

Laugksch, RC (2000). Scientific literacy: A conceptual overview. Science Education, 84, 71-94.

Landau, L.D. (1991) Física Teórica: Mecánica. Barcelona: Ed. Reverte.

Manzanilla, H. y Falcon, N. (Octubre de 2013) Comparación Epistémica de la Curricula En Física, entre los Pensa de Secundaria de Chile, Ecuador, México y Venezuela, Acta Científica. I Congreso de Redes de Investigación: Región Central. UPEL Maracay.

Manzanilla, H. y Falcon, N. (2014). Comparación Curricular entre los Pensa de estudio de Chile y Venezuela. Criterios Epistemológicos en Física en nivel medio. Recuperado de: https://issuu.com/umce/docs/extramuros2014

Morín, E. (1999) Los siete saberes necesarios para la educación del futuro. UNESCO. Paris: Edit Santillana.

Newton, I. (1687) Principios matemáticos de la filosofía natural. Madrid: Alianza Editorial.

OCDE (2009). PISA 2009. Assessment framework-key competencies in reading, mathematics and science. Paris: OCDE.

Orellana-Sepúlveda, C., Quintanilla-Gatica, M., Páez-Cornejo, R. (2018) Concepciones sobre enseñanza y aprendizaje de las Ciencias Naturales de educadoras de párvulos en formación en Chile y sus relaciones con modelos de racionalidad científica. Ciênc. Educ, 24, 1029-1041

Rivas, A. y Sanchez, B. (2016). Políticas y resultados educativos en América Latina: un mapa comparado de siete países (2000-2015). Relieve, 22, 1-30. 
Rodríguez, M.E (2017) Currículum, educación y cultura en la formación docente del siglo XXI desde la complejidad. Educación y Humanismo, 19, 425-440.

Ruiz , F. (2007) Modelos didácticos para la enseñanza de las ciencias naturales. Revista Latinoamericana de Estudios Educativos, 3, 41 - 60

Ruiz, M. C., Schoo, S. (2014). La obligatoriedad de la educación secundaria en América Latina. Convergencias y divergencias en cinco países. Foro de Educación, 12, 71-98.

Sasseron, L.,Carvalho, A.M. (2011) Alfabetização Científica: uma revisão bibliográfica. Investigações em Ensino de Ciências, 16, 59-77.

Valenzuela, A. (2004) Opciones Epistemológicas y diseño curricular: Referencias para el curriculum de formación de profesores. Revista Perspectiva Educacional, 44, 37-69

Thagard, P. (1992). Conceptual revolutions. Princeton University Press.

Whewell, W. (1840) The Philosophy of the Inductive Sciences: Founded Upon Their History, Volumen 1. London: J. Parker Ed.

Whewell, W. (1847) History of the Inductive Sciences. London: J. Parker Ed.

Recibido: 13.11.2019 Aceptado: 29.03.2020 